Document downloaded from:

http://hdl.handle.net/10251/38427

This paper must be cited as:

Ricolfe Viala, C.; Sánchez Salmerón, AJ.; Valera Fernández, A. (2013). Efficient lens distortion correction for decoupling in calibration of wide angle lens cameras. IEEE Sensors Journal. 13(2):854-863. doi:10.1109/JSEN.2012.2229704.

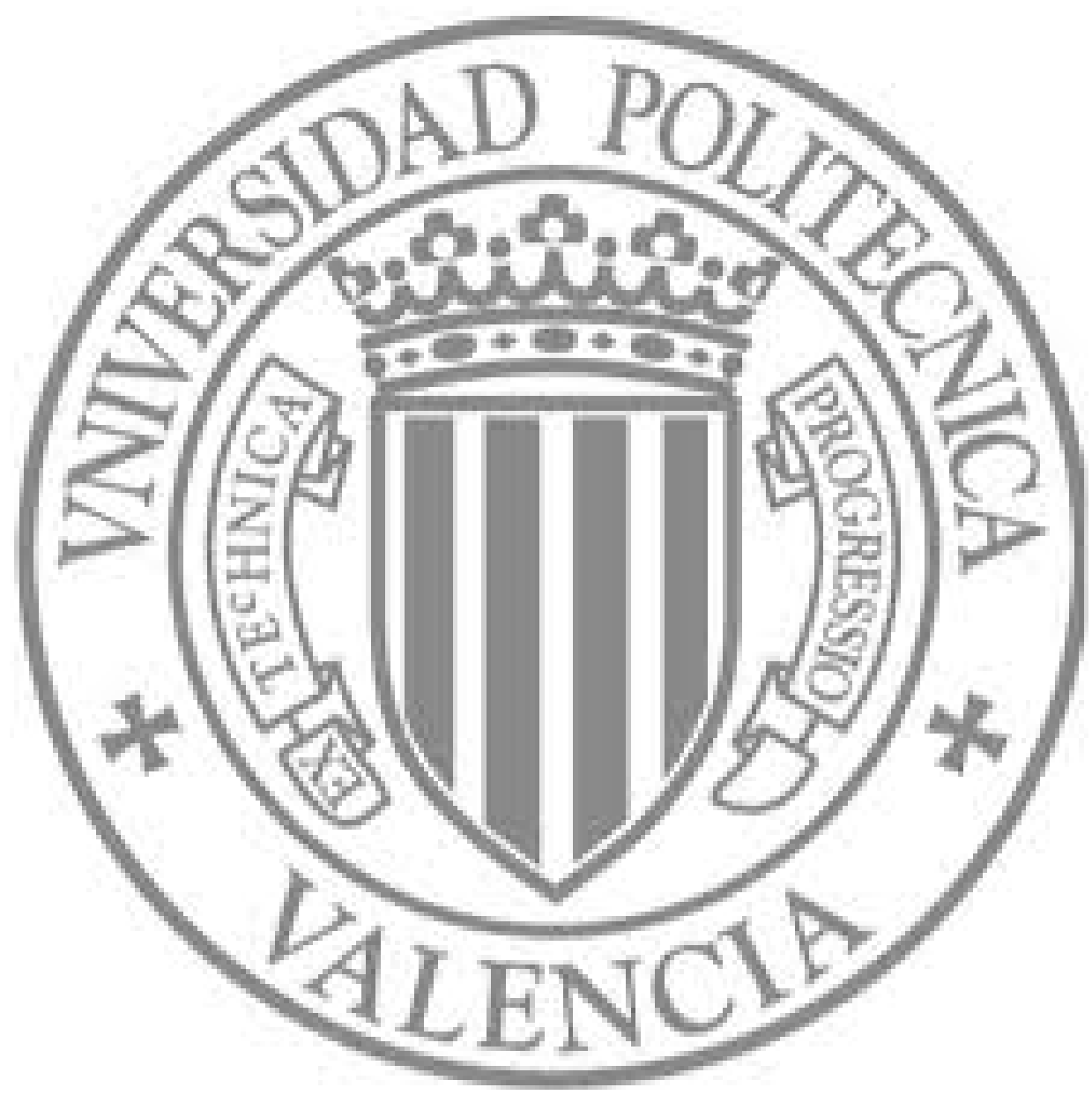

The final publication is available at

http://ieeexplore.ieee.org/xpl/articleDetails.jsp?arnumber=6359740

Copyright Institute of Electrical and Electronics Engineers (IEEE) 


\title{
Efficient Lens Distortion Correction for Decoupling in Calibration of Wide Angle Lens Cameras
}

\author{
Carlos Ricolfe-Viala, Member, IEEE, Antonio-Jose Sanchez-Salmeron and Angel Valera
}

\begin{abstract}
In photogrammetry applications, camera parameters must be as accurate as possible to avoid deviations in measurements from images. Errors increase if wide angled lens cameras are used. Moreover, the coupling between intrinsic and extrinsic camera parameters and the lens distortion model influences the result of the calibration process notably. This paper proposes a method for calibrating wide angle lens cameras which takes into account the existing hard coupling. The proposed method obtains stable results which do not depend on how the image lens distortion is corrected.
\end{abstract}

Index Terms - close-range photogrammetry, camera calibration, lens distortion, distortion model, metric calibration, robust estimators

\section{INTRODUCTION}

$\mathrm{G}$ EOMETRIC camera calibration consist of computing the $\mathbf{J}_{\text {mapping between points in the scene and their }}$ corresponding points in the image. This mapping is basically represented with the pin-hole model, composed with intrinsic and extrinsic parameters. Extrinsic parameters represent the transformation between the scene and camera coordinates systems and intrinsic parameters give the projective transformation of points in the scene to points in the image. If image is distorted the mapping between both set of points is improved if distortion is corrected previously. Lens distortion is corrected by using a lens distortion model which transforms distorted pixels locations in the image to corrected ones. When camera is calibrated both pin-hole and lens distortion models are computed.

Manuscript received November 1, 2011. This work was partially funded by the Universidad Politécnica de Valencia research funds (PAID 2010-2431 and PAID 10017), Generalitat Valenciana (GV/2011/057) and by Spanish government and the European Community under the project DPI2010-20814C02-02 (FEDER-CICYT) and DPI2010-20286 (CICYT).

C. Ricolfe-Viala is with the Instituto de Automática e Informática Industrial of Universitat Politècnica de València, Valencia, 46022, Spain. Email: cricolfe@isa.upv.es.A.J. Sánchez-Salmerón is with the Instituto de Automática e Informática Industrial of Universitat Politècnica de València, Valencia, 46022, Spain. E-mail: asanchez@isa.upv.es.

A. Valera is with the Instituto de Automática e Informática Industrial of Universitat Politècnica de València, Valencia, 46022, Spain. E-mail: giuprog@isa.upv.es.
Extrinsic and intrinsic camera pin-hole model parameters present high correlation. This means that several models can represent accurately the mapping between points in the scene, if a reprojection error is used for validating it. Moreover, lens distortion correction influences the computed pin-hole model notably. Fig. 5 shows a distorted image which has been corrected using two of existing lens distortion correction methods. Depending on which image is used to calibrate the pin-hole model, results are different since calibration data is different obviously. These differences are reflected in the calibrated camera parameters. If a wide angle lens camera is calibrated, variations increase significantly since images are high distorted.

To represent low distortion in the image, Brown [1] proposed the radial, decentring and prism distortion model which has been widely used [2]-[4]. With high distortion, higher order terms of radial, decentering and prism distortion do not fully represent the camera lens distortion [4]-[6]. Therefore, to represent the high distortion effect in the image Basu and Licardie [7] proposed the logarithmic distortion model. Also, Devernay and Faugeras [4] introduced the fieldof-view distortion model for fish-eye lenses. Fitzgibbon [8] recommends the use of the division model to express high distortion with just one parameter. Hartley and Kang in [9] propose the nonparametric model which only considers radial distortion but compensates distortion in all types of lenses, from standard low-distortion to high distortion fish-eye lenses. Claus and Fitzgibbon [10] proposed a lifting strategy to build the rational function distortion model for a wide range of highly distorted cameras.

Normally existing camera calibration methods compute the pin-hole and lens distortion in a two step process where first lens distortion is corrected and second pin-hole is computed [11-16]. Other methods compute both distortion and pin-hole models in one step [17-18]. Any described method do not consider the coupling of pin-hole and lens distortion models except in [19], where the coupling between intrinsic and extrinsic camera parameters is taken into account but the coupling with the distortion model is not considered.

In this work we propose a calibration process in which the coupling between pin-hole intrinsic and extrinsic camera parameters and lens distortion models is considered. To avoid the coupling between both models, locations of pixels which 
are going to participate in the calibration process are corrected previously. Using the basic mapping projection functions and a set of constraints which define the image formation, pixel locations in the image are corrected before pin-hole and lens distortion models are computed. The result is the pin-hole and the lens distortion model which represent the calibrated camera really.

Paper is organized as follow. First, the coupling between intrinsic, extrinsic and lens distortion models is briefly explained. Second, the pixel location correction method and the calibration process are described. Third, experimental results are presented. Paper ends with some concluding remarks.

\section{THE COUPLING BETwEEN INTRINSIC, EXTRINSIC AND LENS DISTORTION MODEL}

Pin-hole model is defined as follows:

$s^{i, a} q_{0}=K \cdot\left[\begin{array}{ll}{ }^{a} R & { }^{a} t\end{array}\right] \cdot{ }^{i} p=K \cdot\left[\begin{array}{llll}{ }^{a} r_{1} & { }^{a} r_{2} & { }^{a} r_{3} & { }^{a} t\end{array}\right] \cdot{ }^{i} p$

where ${ }^{i, a} \boldsymbol{q}_{\boldsymbol{o}}$ represent the image coordinates of a point ${ }^{i} \boldsymbol{p}$ in the scene. $\left[{ }^{a} \boldsymbol{R}{ }^{a} t\right]$ is a $3 \times 4$ matrix containing the extrinsic parameters which transform both the world and the camera coordinate systems (rotation matrix ${ }^{\boldsymbol{a}} \boldsymbol{R}$ and translation vector $\left.{ }^{a} t=\left(t_{\mathrm{x}}, t_{\mathrm{y}}, t_{\mathrm{z}}\right)^{\prime}\right)$. $a$ represents one camera location. $\boldsymbol{K}$ is a $3 \mathrm{x} 3$ matrix with the camera intrinsic parameters.

$$
K=\left[\begin{array}{ccc}
\alpha_{u} / f & \gamma & u_{0} \\
0 & \alpha_{v} / f & v_{0} \\
0 & 0 & 1
\end{array}\right]
$$

with $\left(u_{0}, v_{0}\right)$ the coordinates of the principal point, $f$ is the focal length (distance from the focal point $F$ to the image plane) and $\alpha_{u}$ and $\alpha_{v}$ the scale factors according to image axes ( $\mathrm{mm}$ to pixels scaling). $\boldsymbol{s}$ is an arbitrary scale factor since coordinates are in the projective space and $\gamma$ is the skew factor. Fig. 5 shows how a distorted image is corrected with two existing methods. Fig. 5(a) shows the original captured distorted image and Figs. 5(g) and 5(j) are corrected using two different lens distortion correction methods. After correction, image of Fig. 5(g) seems closer to the camera than image of Fig. 5(j). However original image is the same in both cases. Which one is the precise image correction? How does this effect influence the calibration of the camera parameters? From the point of view of the camera parameters, this effect is reflected in the focal length $f$ and in the translation in the $Z_{c}$ axis. Fig. 1 shows this influence as follow. Without lose of generality, we assume an ideal camera where $\alpha_{u}=1, \alpha_{v}=1$ and $\gamma=0, r_{u}$ is the radius of the point location ${ }^{i} \boldsymbol{q}_{\boldsymbol{o}}$ in the image without distortion of the projected point ${ }^{i} \boldsymbol{p}$ with radius $r_{i}$. $f$ and $t_{\mathrm{z}}$ represent the exact camera parameters which are computed when camera is calibrated using $r_{u}$ and $r_{i}$. If a distorted image is captured, lens distortion is corrected and $r_{c}$ is the radius in the corrected image of the projected point $r_{i}$. Camera

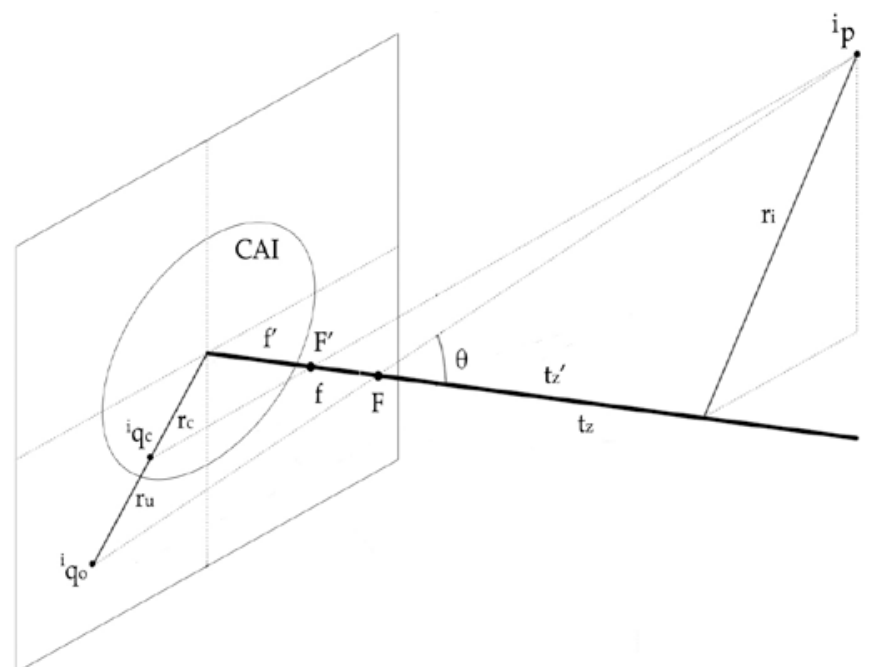

Fig. 1 . The projection in the image plane ${ }^{i} q_{o}$ of a point in the scene ${ }^{i} p$ is defined with the pin-hole model. Also, the rectilinear projection function describes the undistorted location in the image plane $r_{u}$ which depends on the incidence angle $\theta$. The variation of radial distance from the centre of the image influences the focal length $f$ and the translation distance $t_{z}$ since both parameters are coupled. If ' $q_{o}$ is moved to ' $q_{c}$, focal length is $f$ ' and the translation distance is $t^{\prime} \mathrm{z}$. The central area of the image (CAI) is defined with an incidence angle of $\theta=25^{\circ}$.

calibration using $r_{c}$ and $r_{i}$, will compute $f^{\prime}, t_{\mathrm{z}}$ ' which does not represent the exact camera parameters. From the point of view of the camera calibration process, results are correct since the computed model represent the transformation between $r_{c}$ and $r_{i}$, accurately. However, the computed parameters are just adapted to the calibration data but they do not represent the calibrated camera not at all. Consequently, lens distortion correction influences the camera calibration seriously and especial care should be taken into account to correct it. Camera should be calibrated with corrected points which are closer to the ideal point locations. If corrected points are deviated from the ideal ones, calibration result will be erroneous.

\section{The Proposed Camera Calibration Process}

The proposed calibration process corrects the pixel location in the distorted image of those pixels which are going to participate in the calibration process. This correction is done before pin-hole and lens distortion is calibrated. When pixels locations are corrected, pin-hole and lens distortion models are calibrated with some of existing methods.

To correct the location of distorted pixels in the image, the basic mapping projection functions and a set of constraints which define the image formation are used. Projective geometry constraints are shown in Fig. 2. Since points in a chessboard are located in equally distanced straight lines which are perpendicular and parallel each other, images of these equally distanced perpendicular and parallel straight lines must fulfil theses constraints under perspective projection. These are the cross-ratio, straight lines, vanishing points, horizon line and focal length constraint. 


\section{A. Cross-ratio}

If $\operatorname{CR}\left(\boldsymbol{p}_{1}, \boldsymbol{p}_{2}, \boldsymbol{p}_{3}, \boldsymbol{p}_{4}\right)$ represents the cross-ratio of four points in the template, this cross-ratio is equal to all four set of points which are equally distributed in the planar template. Points in the template are separated in $n$ sets of $m$ points which form straight lines, where $n$ is the number of straight lines in the calibration template (horizontals and verticals) and $m$ is the number of points in each line. So, $\boldsymbol{q}^{\boldsymbol{k}, \boldsymbol{l}}$ is a point $k$ of the straight line $l$ in one image, $l=1 . . n, k=1 . . m$, the following index measures how a set of points are equally separated under perspective projection.

$J_{C R}=\sum_{l=1}^{n} \sum_{k=1}^{m-3}\left(C R\left(q^{k, l}, q^{k+1, l}, q^{k+2, l}, q^{k+3, l}\right)-C R\left(p_{1}, p_{2}, p_{3}, p_{4}\right)\right)^{2}$

Taking into account that the chosen planar template has straight lines in vertical and horizontal directions, this index must be true for horizontal and vertical straight lines. Each point detected in the image ${ }^{i, a} \boldsymbol{q}_{\boldsymbol{d}}$ belongs to two lines and it represents the intersection. Thus, the cross-ratio of the point ${ }^{i, a} \boldsymbol{q}_{d}$ with all its neighborhoods must be true in both directions. $\mathrm{CR}\left(\boldsymbol{p}_{1}, \boldsymbol{p}_{2}, \boldsymbol{p}_{3}, \boldsymbol{p}_{4}\right)$ is computed previously when the planar template is designed.

\section{B. Straight lines}

On the other hand, since points in the image have been separated in $n$ straight lines of $m$ points, they must fit in the lines perfectly. So, if a set of points $q^{k, l}=\left(u^{k, l}, v^{k, l}, w^{k, l}\right)$ fits in a straight line, the following expression is true:

$$
\left[\begin{array}{ccc}
u^{1, l} & v^{1, l} & w^{1, l} \\
u^{2, l} & v^{2, l} & w^{2, l} \\
\ldots & \ldots & \ldots \\
u^{m, l} & v^{m, l} & w^{m, l}
\end{array}\right] \cdot\left[\begin{array}{l}
a_{l} \\
b_{l} \\
c_{l}
\end{array}\right]=\left[\begin{array}{l}
0 \\
0 \\
0 \\
0
\end{array}\right]
$$

where $k=1 . . m$ and $a_{l}, b_{l}, c_{l}$ represent the set of parameters which defines the straight line $l$. In a matrix from we have $\boldsymbol{A} \cdot \boldsymbol{l}=\mathbf{0}$, where $\boldsymbol{A}$ is a $m \times 3$ matrix and $\boldsymbol{l}=\left(a_{l}, b_{l}, c_{l}\right)^{T}$. If line parameters are computed with the $m$ points which belong to the line $\boldsymbol{l}$, the orthogonal distance of the $m$ points to the line $\boldsymbol{l}$ must be zero. Following equation measures how a set of points fit in lines.

$$
J_{S T}=\sum_{l=1}^{n} \sum_{k=1}^{m} d\left(q^{k, l}, l\right)=\sum_{l=1}^{n} \sum_{k=1}^{m} \frac{\left|a_{l} \cdot u^{k, l}+b_{l} \cdot v^{k, l}+c_{l} \cdot w^{k, l}\right|}{\sqrt{a_{l}^{2}+b_{l}^{2}}}
$$

Again, vertical and horizontal straight lines are taken into account.

\section{Vanishing points}

It is well known that parallel lines in the scene will meet in a vanishing point in an image. Also, all vanishing points in one image fit in the horizon line since we have an image of a

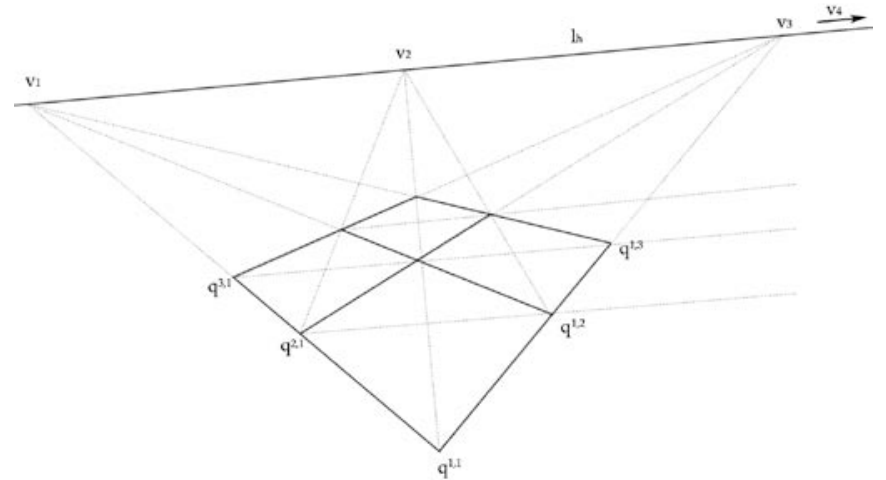

Fig. 2. Perspective projective constraints used for correcting points detected in the image. Cross ratio guaranties that parallel lines remain parallels under perspective projection. Points are corrected to belong to straight lines. All parallel lines meet in a vanishing point in an image. All vanishing points form the horizon line.

planar template. Therefore, straight lines which are parallel in the calibration template will intersect in a unique vanishing point in the image. If the template has horizontal, vertical and both diagonals parallel lines, four vanishing points exist in any template image. Moreover, these four vanishing points should fit in a horizon line perfectly if projective transformations are taken into account. These constrains are used in the point correction process.

If $\boldsymbol{a}_{\boldsymbol{l}}, \boldsymbol{b}_{\boldsymbol{l}}, \boldsymbol{c}_{\boldsymbol{l}}$ represent the set of parameters which defines the straight line $l$ in the image, following expression defines the vanishing point in the image for a set of lines.

$\left[\begin{array}{ccc}a_{1} & b_{1} & c_{1} \\ a_{2} & b_{2} & c_{2} \\ \ldots & \ldots & \ldots \\ a_{t} & b_{t} & c_{t}\end{array}\right] \cdot\left[\begin{array}{l}u_{v p} \\ v_{v p} \\ w_{v p}\end{array}\right]=\left[\begin{array}{l}0 \\ 0 \\ 0 \\ 0\end{array}\right]$

$l=1 . . t$ where $t$ represent the number of parallels lines and $\boldsymbol{q}_{v p}=\left(u_{v p}, v_{v p}, w_{v p}\right)$ is the vanishing point in homogeneous coordinates. Expressed in a matrix form we have:

$B \cdot q_{v p}=0$

Since an image has four set of parallels lines, ${ }^{1} \boldsymbol{q}_{v p},{ }^{2} \boldsymbol{q}_{v p},{ }^{3} \boldsymbol{q}_{v p}$, ${ }^{4} \boldsymbol{q}_{v p}$ represent the four corresponding vanishing points in an image which are computed with the eigen vector of matrix $\boldsymbol{B}$. Following function should be zero if all parallel straight lines intersect in the corresponding vanishing point.

$J_{V P}=\sum_{i=1}^{4} \sum_{l=1}^{t} d\left({ }^{i} q_{v p}, l_{l}\right)$

\section{Horizon Line}

The orthogonal distance of a vanishing point to all parallel straight lines is zero. If these four vanishing points fit in the horizon line, the following expression is true. 
$\left[\begin{array}{c}{ }^{1} q_{v p}^{T} \\ { }^{2} q_{v p}^{T} \\ { }^{3} q_{v p}^{T} \\ { }^{4} q_{v p}^{T}\end{array}\right] \cdot\left[\begin{array}{l}a_{h} \\ b_{h} \\ c_{h}\end{array}\right]=\left[\begin{array}{l}0 \\ 0 \\ 0 \\ 0\end{array}\right]$

where $\boldsymbol{I}_{\boldsymbol{h}}=\left[\boldsymbol{a}_{\boldsymbol{h}}, \boldsymbol{b}_{\boldsymbol{h}}, \boldsymbol{c}_{\boldsymbol{h}}\right]$ represents the set of parameters which defines the horizon line computed as an eigen vector of matrix C. Matrix $\boldsymbol{C}$ is formed with the four vanishing points coordinates. According to perspective projection constraints all vanishing points fit in the horizon line perfectly. Following expression must be true:

$J_{H L}=\sum_{i=1}^{4} d\left({ }^{i} q_{v p}, l_{h}\right)$

\section{E. Focal Length}

Proposed previous constraints are true for any image of the template alone. If several images are captured with the same camera, points should be corrected taking into account constrains concerning the camera intrinsic parameters. If we keep the principal point fixed at the center of the image, and assume orthogonal axis, intrinsic parameters matrix $\boldsymbol{K}$ contains only the focal lengths $f_{x}$ and $f_{y}$.

$K=\left[\begin{array}{ccc}1 / f_{x} & 0 & 0 \\ 0 & 1 / f_{y} & 0 \\ 0 & 0 & 1\end{array}\right]$

Revising [14-16] works, the four vanishing points ${ }^{1} \boldsymbol{q}_{v p},{ }^{2} \boldsymbol{q}_{v p}$, ${ }^{3} \boldsymbol{q}_{v p},{ }^{4} \boldsymbol{q}_{v p}$, provide two scalars constrains in the focal lengths $f_{x}$ and $f_{y}$, since they are the vanishing points of mutually orthogonal lines in the scene. These two scalars constrains are:

$\frac{{ }^{1} u_{v v} \cdot{ }^{2} u_{v p}}{f_{x}^{2}}+\frac{{ }^{1} v_{v p} \cdot{ }^{2} v_{v p}}{f_{y}^{2}}+{ }^{1} w_{v p} \cdot{ }^{2} w_{v p}=0$

$\frac{{ }^{3} u_{v p} \cdot{ }^{4} u_{v p}}{f_{x}^{2}}+\frac{{ }^{3} v_{v p} \cdot{ }^{4} v_{v p}}{f_{y}^{2}}+{ }^{3} w_{v p} \cdot{ }^{4} w_{v p}=0$

where ${ }^{1} u_{v p},{ }^{1} v_{v p},{ }^{1} w_{v p},{ }^{2} u_{v p},{ }^{2} v_{v p},{ }^{2} w_{v p},{ }^{3} u_{v p},{ }^{3} v_{v p},{ }^{3} w_{v p},{ }^{4} u_{v p},{ }^{4} v_{v p}$, ${ }^{4} w_{v p}$, are the known pixel coordinates of the vanishing points

${ }^{1} \boldsymbol{q}_{v p},{ }^{2} \boldsymbol{q}_{v p},{ }^{3} \boldsymbol{q}_{v p},{ }^{4} \boldsymbol{q}_{v p}$ respectively.Expressed in a matriz form we have:

$$
\left[\begin{array}{ll}
{ }^{1} u_{v p} \cdot{ }^{2} u_{v p} & { }^{1} v_{v p} \cdot{ }^{2} v_{v p} \\
{ }^{3} u_{v p} \cdot{ }^{4} u_{v p} & { }^{3} v_{v p} \cdot v_{v p}
\end{array}\right] \cdot\left[\begin{array}{l}
1 / f_{x}^{2} \\
1 / f_{y}^{2}
\end{array}\right]=-\left[\begin{array}{l}
{ }^{1} w_{v v} \cdot{ }^{2} w_{v p} \\
{ }^{3} w_{v p} \cdot{ }^{4} w_{v p}
\end{array}\right]
$$

Given several images captured with the same camera, the camera focal length can be computed as a least squares minimization problem as $\boldsymbol{f}=-\left(\boldsymbol{D}^{\mathrm{T}} \cdot \boldsymbol{D}\right)^{-1} \cdot \boldsymbol{D}^{\mathrm{T}} \cdot \boldsymbol{E}$ where:

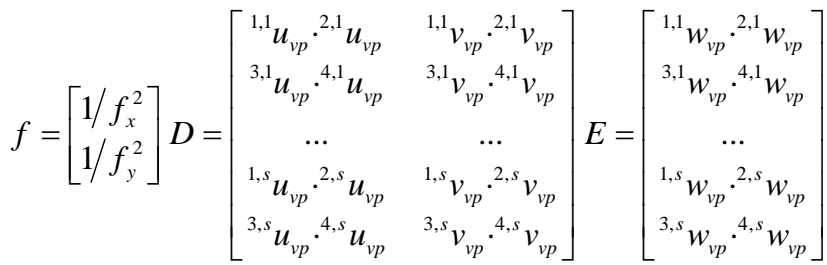

${ }^{i, a} \boldsymbol{q}_{v p}=\left({ }^{i, a} u_{v p},{ }^{i, a} v_{v p},{ }^{i, a} w_{v p}\right)$ represents the vanishing point $i$, in the image $a$. $i=1 . .4$ and $a=1$..s where $s$ is the number of images. If the four vanishing points in all images are defined with the same camera, following function is zero:

$J_{F L}=G^{T} \cdot G$

where $\boldsymbol{G}=-\boldsymbol{D} \cdot\left(\boldsymbol{D}^{\mathrm{T}} \cdot \boldsymbol{D}\right)^{-1} \cdot \boldsymbol{D}^{\mathrm{T}} \cdot \boldsymbol{E}+\boldsymbol{E} . \boldsymbol{G}$ contains the error of the focal length computed with a given set of vanishing points. The minimization principle of least square method (LSM) has been used.

\section{F. Defining the scale factor}

Proposed constraints are true if points are scaled in the image. Control points of images of Figs 5(g) and 5(j) could satisfy all proposed constraints. Therefore it is necessary a scale factor which defines the exact pixel location in the image. To define the exact scale factor mapping projection functions are used. Since, lens manufacturers attempt to design lenses which respond to any mapping projection function, the mapping projection function will help us to decide how the correction should be done. Several mapping projection functions exist depending on the field of view of the lens.

The rectilinear projection function preserves the linearity and is distortion free. Rectilinear projection is also called pinhole and is given as:

$r_{u}=f \cdot \tan (\theta)$

If wide field of view lens is necessary, the size of the projected image becomes very large under rectilinear projection. A field of view of $180^{\circ}$ will need an image of infinite size. For wide angled lens other projection functions exist such as equidistant, orthographic, equisolid or stereographic. The equidistant projection function computes the distance on the image plane $r_{q}$ proportional to the angle in radians of the incident ray as follows:

$r_{q}=f \cdot \theta$

The orthographic projection function maps the incident ray in the image as:

$r_{o}=f \cdot \sin (\theta)$

The equisolid projection function is also known as equal- 
area and is defined as:

$r_{e}=2 \cdot f \cdot \sin (\theta / 2)$

The stereographic projection function is:

$r_{s}=2 \cdot f \cdot \tan (\theta / 2)$

In all cases, $f$ is the distance between the focal point and the image plane, $\theta$ is the incidence angle of the projected ray to the optical axis of the camera and $r_{u}, r_{q}, r_{o}, r_{e}$ and $r_{s}$, is the distance from the focal point on the image plane. Equidistant, orthographic, equisolid and stereographic projection functions distort images differently, and the manner of distortion is referred to as their mapping function. Images from a wide angle or fish-eye lens camera have significant radial lens distortion where points on the image plane are displaced from their ideal position along a radial axis from the distortion centre.

The rectilinear projection function defines the right focal length of a lens only. If camera is calibrated with pixels which satisfy the rectilinear projection function, the computed focal length will be correct. Therefore, the focal length of a lens constructed under the equidistant, orthographic, equisolid and stereographic projection functions will be defined with the part of the lens which has a performance similar to the rectilinear projection function. If we find an area in the image plane where any wide angle mapping projection functions have similar performance than the rectilinear projection function, all pixels in this area will give the correct focal length of a wide angle lens if they are used in the calibration process. If we use pixels located in another part of the image to calibrate the camera, the computed focal length will be erroneous.

Comparing all mapping projection functions, projected ray is the same $r_{u}=r_{q}=r_{o}=r_{e}=r_{s}$ when $\theta=0$. To obtain an area in the image plane, a nonzero incidence angle $\theta$ is needed. A nonzero angle $\theta$ is computed if a deviation between rectilinear $r_{u}$ and distorted $r_{d}$ projection is accepted. Considering a $10 \%$ of deviation between both radial distances, an incidence angle $\theta=25$ degrees gives a difference between both radial distances under $10 \%$. Therefore, radial distances in the distorted image with an incidence angle $\theta$ under 25 degrees can be used to obtain the focal length $f$ of the wide angle lens. This value is computed using the rectilinear and orthographic projection functions assuming that $r_{o}=0.9 \cdot r_{u}$.

$$
0.9 \cdot f \cdot \tan (\theta)=f \cdot \sin (\theta)
$$$$
\theta=\arccos (0.9) \approx 25^{\circ}
$$

If equidistant, equisolid or stereographic projection functions are used, similar value of $\theta$ is computed. We call the central area of the image (CAI), the area of the image which has an incidence angle under $\theta=25^{\circ}$. Fig. 1 shows this CAI area. Since CAI could represent the ninth part of the image or less, calibration data will be reduced notably. To increase the

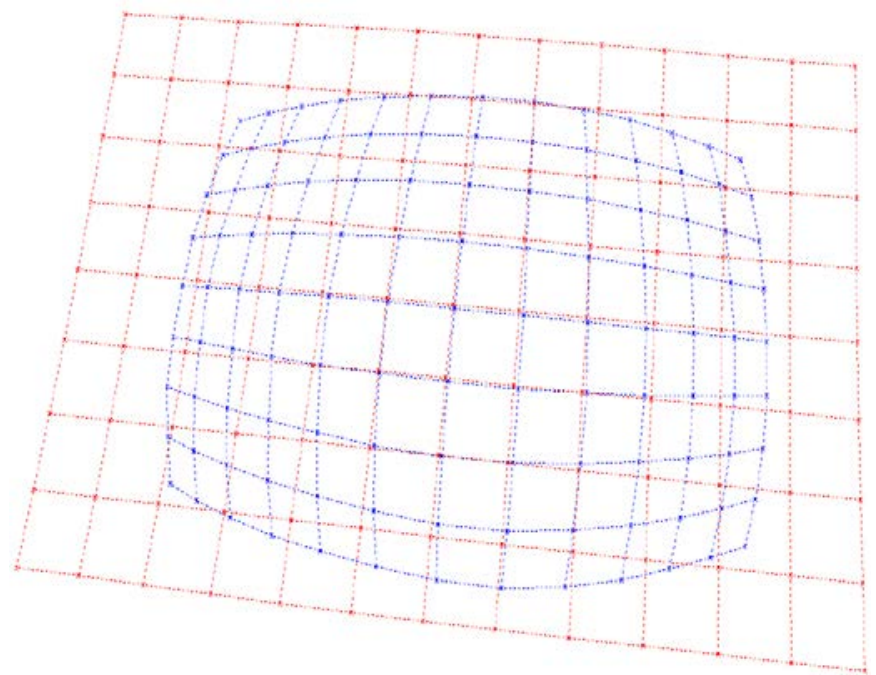

Fig. 3. Blue dots represent detected points ${ }^{i, a} \boldsymbol{q}_{d}$ in the distorted image of Fig. 5(a). Red dots represent undistorted points ${ }^{i, a} \boldsymbol{q}_{\boldsymbol{o}}$ corrected with the proposed method. Pixels located in the central area of the image are not moved while pixels located in the border of the distorted image are moved to accomplish all projective geometry constraints.

calibration data, we propose to correct all remaining pixels in the image using the location of pixels in CAI assuming that an image of a known calibration template is used to calibrate the camera. If a chessboard is used as a calibration template, points in the image plane which are in CAI will be located correctly and the remaining points can be corrected according with the location of the points in CAI. To correct the remaining points in the image, projective geometry constraints which define the image formation of a chessboard are used.

\section{G. Distorted pixel location correction}

Joining all proposed geometrical constraints, the following equation measures how a set of undistorted points of one or several images, correspond to the points of a "chessboard" template under perspective projection rules.

$J=J_{C R}+J_{S T}+J_{V P}+J_{H L}+J_{F L}$

The non linear minimization has as inputs the cross ratio value of the template points $\operatorname{CR}\left(\boldsymbol{p}_{1}, \boldsymbol{p}_{2}, \boldsymbol{p}_{3}, \boldsymbol{p}_{4}\right)$, locations of points in CAI area and the set of points in the image or images ${ }^{i, a} \boldsymbol{q}_{d}$. For a given set of points, straight lines parameters $\left(a_{l}, b_{l}\right.$, $c_{l}$ ), vanishing points ${ }^{1, j} \boldsymbol{q}_{\boldsymbol{v} p},{ }^{2, j} \boldsymbol{q}_{\boldsymbol{v} \boldsymbol{p}},{ }^{3, j} \boldsymbol{q}_{\boldsymbol{v} \boldsymbol{p}},{ }^{4, j} \boldsymbol{q}_{\boldsymbol{v} \boldsymbol{p}}$, horizons lines ${ }^{\boldsymbol{j}} \boldsymbol{I}_{h}$, and focal lengths $f_{x}, f_{y}$ are computed. With the computed parameters, equation (28) is evaluated and points locations ${ }^{i, \boldsymbol{a}} \boldsymbol{q}$ are corrected to minimize (28). This process is repeated until a minimum is reached. When (28) is minimized, distorted points extracted from the image ${ }^{i, a} \boldsymbol{q}_{\boldsymbol{d}}$ have been undistorted to ${ }^{\boldsymbol{i}, \boldsymbol{a}} \boldsymbol{q}_{\boldsymbol{o}}$. Fig 3 shows distorted points ${ }^{i, \boldsymbol{a}} \boldsymbol{q}_{\boldsymbol{d}}$ in blue and corrected ones ${ }^{i, a} \boldsymbol{q}_{\boldsymbol{o}}$ in red. Points located in the CAI area are not moved. A Levenberg-Marquardt non linear minimization algorithm is used which starts with the set of points in the image or images ${ }^{i, a} \boldsymbol{q}_{d}$ and ends with the set of undistorted points ${ }^{i, \boldsymbol{a}} \boldsymbol{q}_{\boldsymbol{o}}$.

To improve the condition of the non-linear minimization process, points coordinates are referred to the center of the 
image and not to the left top corner. If point's coordinates are referred to the center of the image, the evaluation of $J_{F L}$ is direct also.

If this non linear minimization process is compared with the non linear minimization step of any calibration method, this process is better conditioned notably. Non linear minimization looks for points coordinates in pixels only. This means that all variables produce equal alteration of error function value when a variation of 1 unit is done with any of them. The non linear minimization step of existing calibration methods looks for values of the principal point, focal lengths and rotation and translation vector parameters. Obviously, a variation of translation vector in 1 meter has different effects in the error function than a variation of principal point value in 1 pixel. With the proposed method, risks of finishing the non linear minimization step in a local minimum decrease. On the contrary the number of variables increases and the minimization time enlarges notably. Since the calibration procedure is an off-line process this fact does not represent any inconvenience.

\section{H. Computing the lens distortion and the pin-hole models}

The connection between corrected points ${ }^{i, a} \boldsymbol{q}_{\boldsymbol{o}}$ and detected ones symbolized with ${ }^{i, a} \boldsymbol{q}_{\boldsymbol{d}}$ is done with the lens distortion model. Several distortion models exist. Revising the lens distortion model evaluation in [20], the rational function lens distortion model presented by Claus and Fitzgibbon in [10] can represent low distortion and high distortion accurately. The logarithmic of Basu and Licardie in [7], the division of Fitzgibbon in [8], and field-of-view of Devernay and Faugeras in [4] distortion models have similar performance. The radial, tangential and prism distortion model does not work properly if high distortion is present.

The rational function model is given by:

$$
d\left(u_{d}, v_{d}\right)=\left[\begin{array}{c}
a_{11} \cdot u_{d}^{2}+a_{12} \cdot u_{d} \cdot v_{d}+a_{13} \cdot v_{d}^{2}+a_{14} \cdot u_{d}+a_{15} \cdot v_{d}+a_{16} \\
a_{21} \cdot u_{d}^{2}+a_{22} \cdot u_{d} \cdot v_{d}+a_{23} \cdot v_{d}^{2}+a_{24} \cdot u_{d}+a_{25} \cdot v_{d}+a_{26} \\
a_{31} \cdot u_{d}^{2}+a_{32} \cdot u_{d} \cdot v_{d}+a_{33} \cdot v_{d}^{2}+a_{34} \cdot u_{d}+a_{35} \cdot v_{d}+a_{36}
\end{array}\right]
$$

It can be expressed in a linear combination of the distortion parameters as:

$d\left(u_{d}, v_{d}\right)=A \cdot x\left(u_{d}, v_{d}\right)$

where $\boldsymbol{d}$ is a vector in the camera coordinates that represents the ray direction along which pixel $\boldsymbol{q}_{d}=\left(u_{d}, v_{d}\right)$ samples. $\boldsymbol{A}$ is a $3 \times 6$ matrix containing distortion parameters and $\boldsymbol{x}$ is 6-vector of monomials in $u_{d}$ and $v_{d}$ which define the lifting of the image point $\left(u_{d}, v_{d}\right)$ to a six dimensional space:

$$
x\left(u_{d}, v_{d}\right)=\left[\begin{array}{llllll}
u_{d}^{2} & u_{d} \cdot v_{d} & v_{d}^{2} & u_{d} & v_{d} & 1
\end{array}\right]^{T}
$$

Undistorted image coordinates $\boldsymbol{q}_{\boldsymbol{o}}=\left(u_{o}, v_{o}\right)$ are computed by the perspective projection of $\boldsymbol{d}\left(u_{d}, v_{d}\right)$ :

$$
q_{o}=\left(u_{o}, v_{o}\right)=\left(\frac{a_{1}^{T} \cdot x\left(u_{d}, v_{d}\right)}{a_{3}^{T} \cdot x\left(u_{d}, v_{d}\right)}, \frac{a_{2}^{T} \cdot x\left(u_{d}, v_{d}\right)}{a_{3}^{T} \cdot x\left(u_{d}, v_{d}\right)}\right)
$$

where rows of $\boldsymbol{A}$ are denoted by $a^{\mathrm{T}}{ }_{1.3 .}$.

Using detected distorted ${ }^{\boldsymbol{i}, \boldsymbol{a}} \boldsymbol{q}_{\boldsymbol{d}}$ points and corrected undistorted ${ }^{i, a} \boldsymbol{q}_{\boldsymbol{o}}$, parameters of the rational function lens distortion model (elements of matrix $\boldsymbol{A}$ ) are computed. Rearranging (30) we have:

$$
\begin{aligned}
& a_{3}^{T} \cdot x\left(u_{i, d}, v_{i, d}\right) \cdot u_{i, 0}=a_{1}^{T} \cdot x\left(u_{i, d}, v_{i, d}\right) \\
& a_{3}^{T} \cdot x\left(u_{i, d}, v_{i, d}\right) \cdot v_{i, 0}=a_{2}^{T} \cdot x\left(u_{i, d}, v_{i, d}\right)
\end{aligned}
$$

which can be expressed in a matrix form as:

$$
\left[\begin{array}{ccc}
-x\left(u_{i, d}, v_{i, d}\right)^{T} & 0 & u_{i, 0} \cdot x\left(u_{i, d}, v_{i, d}\right)^{T} \\
0 & -x\left(u_{i, d}, v_{i, d}\right)^{T} & v_{i, 0} \cdot x\left(u_{i, d}, v_{i, d}\right)^{T}
\end{array}\right] \cdot\left[\begin{array}{c}
a_{1} \\
a_{2} \\
a_{3}
\end{array}\right]=0
$$

Given $n \cdot m$ points, we can stack all equations together to obtain a total of $2 \cdot n \cdot m$ equations or in matrix form as $\boldsymbol{W} \cdot \boldsymbol{a}=\mathbf{0}$, where $\boldsymbol{a}=\left[a_{11}, a_{12}, a_{13}, a_{14}, a_{15}, a_{16}, a_{21}, a_{22}, a_{23}, a_{24}, a_{25}, a_{26}\right.$, $\left.a_{31}, a_{32}, a_{33}, a_{34}, a_{35}, a_{36}\right]^{\mathrm{T}}$. The solution is given by the eigenvector associated with the small eigenvalue of matrix $\boldsymbol{W}$. To refine this solution through maximum likelihood inference, the following error function is minimized:

$\left.J_{R T}=\sum_{i=1}^{n \cdot m}\left(\left\|u_{i, 0}-\frac{a_{1}^{T} \cdot x\left(u_{d}, v_{d}\right)}{a_{3}^{T} \cdot x\left(u_{d}, v_{d}\right)}\right\|+\left\|v_{i, 0}-\frac{a_{2}^{T} \cdot x\left(u_{i, d}, v_{i, d}\right.}{a_{3}^{T} \cdot x\left(u_{i, d}, v_{i, d}\right.}\right\|\right)\right)$

(29) is a non-linear minimization problem solved with the Levenberg-Marquart algorithm. Initial guess of matrix $\boldsymbol{A}$ is obtained by using the closed-form solution and $c_{u}, c_{v}$ is initialized with the principal point. The non-linear minimization always converges to a solution also solving the distortion centre.

To compute the pin-hole model, method proposed by Zhang in [9] is used since this method computes the camera parameters if one or several images of a planar calibration template have been taken. In this case, calibration template points ${ }^{i} \boldsymbol{p}$ and undistorted corrected points ${ }^{i, a} \boldsymbol{q}_{\boldsymbol{o}}$ are used (see [9] for details of the calibration method).

\section{Degenerate configurations}

In general, the proposed algorithm for points location correction obtains good results. However if images are taken under specific conditions, points locations cannot be corrected accurately. This case is when images of the planar template are taken with the camera in a zenital location, straight lines in the image are parallel and therefore they will not meet in any vanishing point. In consequence, since the vanishing points do not exist, the horizon line disappears. As a result constraints 

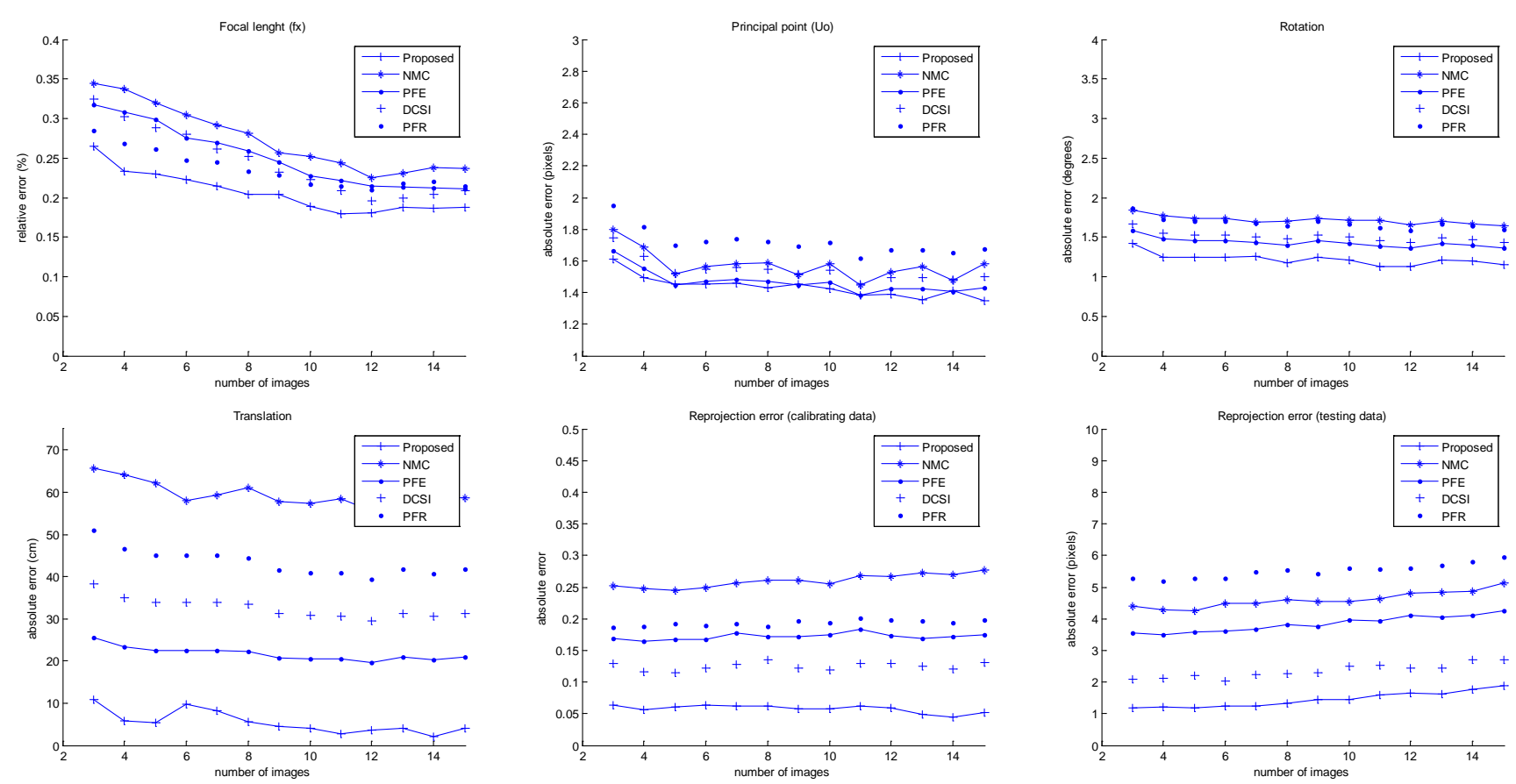

Fig. 4. Result with simulated data varying the number of images. The translation vector varies depending on the lens distortion correction method. Reprojection error is small. This means that the mapping from template points to distorted image points is correct for calibrating data. However translation vector varies since calibration data is different since the lens distortion correction is different depending on the chosen method.

described in subsections $\mathrm{C}, \mathrm{D}$ and $\mathrm{E}$ do not exist and the point location correction algorithm cannot be used as it has been described in this subsection. Taking in to account that camera locations from where images are taken are free defined, zenital location should be avoided if the proposed algorithm is wanted to be used successfully.

Also, since the calibration process is based on a planar template, special care should be taken into account when the calibration template is built. Obviously, any deformation in the planar calibration template will perturb the computed parameters.

From the point of view of the camera parameters calibration algorithm, image plane of several images should not be parallel to provide more constraints on the camera intrinsic parameters (see [9] for details).

\section{EXPERIMENTAL RESULTS}

The proposed calibration method is tested with real and simulated data. The proposed method is compared also with four existing lens distortion correction methods, the non metric calibration of lens distortion (NMC) proposed by Ahmed in [21], the polynomial fish-eye lens distortion correction (PFE) presented by Devernay and Faugeras in [4], the lens distortion correction from a single image (DCSI) presented in [16] by González-Aguilera et al. and the parameter-free radial distortion correction (PFR) proposed by Hartley and Kang in [9]. With these five methods, lens distortion is calibrated and images are undistorted. Each calibration method computes a different distortion model named as $\boldsymbol{D}_{m}$ in equation (36). The NMC computes the traditional radial, decentering and prism lens distortion model. The PFE computes a set of coefficients of a polynomial which do not have the radial or decentering meaning. The DCSI computes two parameters $k_{1}$ and $k_{2}$ which do not have the radial meaning. The PFR determines the radial distortion in a parameter-free way, not relying on any particular radial distortion model. The parameter free feature makes the method useful for fish-eye, wide angle, and narrow angle lenses. After, the pin-hole is calibrated with the method proposed by Zhang in [3] using corrected images. The calibration template is a $210 \mathrm{~mm} \times 297 \mathrm{~mm}$ checkerboard with 165 corners points (11x15), similar to that shown in Fig. 5. Images are taken from 20 different locations. 15 images are used to compute parameters and 5 to test results. Results are evaluated comparing the computed camera parameters values with the simulated or physical ones and evaluating the calibration error. Calibration error is given with the wellknown reprojection error.

$$
\sum_{a} \sum_{i}\left({ }^{i, a} q_{d}-{ }^{i, a} q_{p}\left(K,{ }^{a} R,{ }^{a} t, D_{m}\right)\right)^{2}
$$

where ${ }^{i, a} \boldsymbol{q}_{\boldsymbol{p}}\left(\boldsymbol{K},{ }^{a} \boldsymbol{R},{ }^{a} \boldsymbol{t}, \boldsymbol{D}_{m}\right)$ is the projection of point ${ }^{i} \boldsymbol{p}$ in image $a$ according to equation (1), followed by distortion according to the computed distortion model $\boldsymbol{D}_{m}$.

\section{A. Computer Simulations}

The simulated camera has an image scale factor $s_{x}=1.5$ and an effective focal length $f=8$, resulting in pixel focal lengths of $f_{x}=750, f_{y}=750$. Image resolution is $640 \times 480$ and deviations 
TABLE I

CAMERA PARAMETERS COMPUTED WITH THE FIVE CALIBRATION METHODS (SIMULATED DATA - 10 IMAGES)

\begin{tabular}{llcccccc}
\hline \hline \multirow{2}{*}{ Camera Parameters } & Proposed & NMC & PFE & DCSI & PFR & Real \\
\hline Pin- & $f_{x}$ & 753.5 & 858.9 & 819.6 & 813.6 & 807.8 & 750.0 \\
hole & $f_{y}$ & 745.7 & 852.8 & 804.1 & 814.5 & 805.4 & 750.0 \\
mod. & $u_{0}$ (pixels) & 321.4 & 299.4 & 314.4 & 325.9 & 318.7 & 318.0 \\
& $v_{0}$ (pixels) & 247.2 & 251.8 & 245.8 & 257.3 & 236.4 & 243.0 \\
& $t_{x}(\mathrm{~mm})$ & 50.4 & 50.9 & 56.2 & 47.9 & 43.9 & 50.0 \\
& $t_{y}(\mathrm{~mm})$ & 189.5 & 188.2 & 185.8 & 185.4 & 192.7 & 190.0 \\
& $t_{z}(\mathrm{~mm})$ & -46.6 & -105.7 & -75.3 & -83.4 & -91.3 & -50.0 \\
& $\Psi$ (deg) & 15.8 & 14.2 & 17.4 & 17.8 & 16.0 & 15.0 \\
& $\theta$ (deg) & 90.5 & 88.1 & 97.5 & 95.2 & 96.7 & 90.0 \\
$\phi(\mathrm{deg})$ & 184.4 & 186.2 & 189.6 & 185.4 & 188.6 & 180.0 \\
Rep. err. train. dat. & 0.053 & 0.252 & 0.182 & 0.127 & 0.197 & \\
Rep. err. testing data & 1.652 & 4.576 & 3.982 & 2.465 & 5.957 & \\
\hline \hline
\end{tabular}

between the CCD and the lenses are represented with the principal point at $(318,243)$ pixels and the skew factor $\gamma=1.09$ equivalent to $89.95^{\circ}$.

Images of the checkerboard are generated according to pinhole model and they are distorted using the stereographic projection function. Gaussian noise of mean 2 pixels and $\sigma=1$ is added. The distortion centre is simulated at $c_{u}=315, c_{u}=267$ pixels.

To test the proposed calibration method, we vary the number of images from 3 to 15 . Table I shows the computed parameters with 10 images. The column named with 'Real' represents the real simulated values. All computed values varying the number of images are shown in Fig. 4. To show errors in each camera parameter and to reduce the number of figures, error in the translation vector is expressed as the mean distance in $\mathrm{cm}$ between the known translation vector and the computed one of all used images. Error of the computed rotation matrix is defined as the mean of the angles in degrees between the line which gives the known orientation and the line which gives the computed orientation.

Reprojection error has similar values with all compared calibration methods. Since reprojection error is close to zero, this means that the mapping from template points ${ }^{i} \boldsymbol{p}$ to detected points in the image ${ }^{i, a} \boldsymbol{q}_{\boldsymbol{d}}$ is correct for calibrating data with all tested methods. However, significant differences exist with the computed camera parameters, especially with the translation vector. The computed translation vector depends on how the lens distortion is corrected. Obviously, since undistorted images vary the distance to the camera, this variation is reflected in the translation vector. This effect is shown in Fig. 5 where undistorted images vary the distance to the camera significantly (chessboard of Fig 5 -d is closer to the camera than the one of Fig 5-j). Clearly, the translation vector computed with control points of Fig 5-d,e,f will be different than the translation vector computed with the control points of Fig 5-j,k,l. This difference is reflected in the Fig 4 in the translation vector graph. Specifically, the absolute error in the translation vector computed with the proposed method is under $10 \mathrm{~cm}$. This result is computed with the control points of Fig
TABLE II

CAMERA PARAmETERS COMPUTED WITH THE FIVE CALIBRATION METHODS (REAL DATA)

\begin{tabular}{llccccc}
\hline \hline \multicolumn{2}{c}{ Camera Parameters } & Proposed & NMC & PFE & DCSI & PFR \\
& & & & & & \\
\hline Pin- & $f_{x}$ & 252.9 & 295.9 & 279.5 & 283.5 & 287.5 \\
hole & $f_{y}$ & 253.6 & 302.8 & 284.2 & 298.6 & 275.6 \\
model & $u_{0}$ (pixels) & 314.9 & 309.7 & 302.8 & 307.8 & 298.5 \\
& $v_{0}$ (pixels) & 261.3 & 248.1 & 257.6 & 252.4 & 237.8 \\
& $t_{x}($ mm) & 104.3 & 109.1 & 106.5 & 105.8 & 101.2 \\
& $t_{y}($ mm) & -86.9 & -83.8 & -84.5 & -87.5 & -79.2 \\
& $t_{z}($ mm) & 76.56 & 145.7 & 105.6 & 135.6 & 125.5 \\
& $\Psi$ (deg) & 170.1 & 174.5 & 175.8 & 171.2 & 169.5 \\
& $\theta$ (deg) & 0.1 & 1.5 & 3.7 & 2.5 & 5.6 \\
& $\phi$ (deg) & 174.9 & 176.5 & 179.4 & 175.6 & 168.2 \\
\multirow{2}{*}{ Rep. error (training data) } & 0.786 & 1.026 & 1.568 & 1.125 & 1.452 \\
Rep. error (testing data) & 1.964 & 2.214 & 2.967 & 2.365 & 2.865 \\
& & & & & & \\
\hline \hline
\end{tabular}

5-d,e,f. On the contrary, the translation vector computed with the control points of Fig 5-j,k,l, obtains an absolute error over $50 \mathrm{~cm}$.

Since translation vector is correlated with the focal length parameter $f_{x}$ as was shown in Fig 1, differences between Fig 5$\mathrm{d}$ and Fig 5-j are reflected in the focal length parameter also. According with the simulated results which are shown in Fig 4 in the graph of 'focal length', relative error in the computed focal length varies from $0.2 \%$ to $0.3 \%$ depending on which control points are used. The principal point $U_{o}$ and the rotation parameter do not vary significantly if different control points are used, since they are not influenced by the distance of the camera to the calibration template. Parameters computed with the proposed method are closer to the real ones since distortion correction does not move points located in the CAI area of the image. Points located in CAI are moved significantly if NMC or PFE are used to correct the lens distortion.

\section{B. Real Data}

We have used an IP camera Axis 212 PTZ with $2.7 \mathrm{~mm}$ lens mounted which gives $85^{\circ}$ field of view. Fig. 5 shows three acquired images of $640 \times 480$ pixels with considerable distortion. Again, 20 images have been taken, 15 to calibrate the camera and 5 to test the calibration results which are shown in table II. Using different calibration methods, computed values of intrinsic and extrinsic camera parameters and the calibration error are compared. Results are quite similar to the simulation step. The computed principal point $U_{o}$ and the rotation parameters have similar values with all tested methods. Also, reprojection error is small with all methods and therefore all calibration results are valid a priori. However, the translation vector changes significantly depending on the lens distortion correction method. A calibration with control points of Fig 5-j,k,l gives a translation in the $\mathrm{Z}$ axe of $145.7 \mathrm{~mm}$ while a calibration with control points of Fig 5-d,e,f obtains $76.56 \mathrm{~mm}$. Obviously, a calibration error exists since the original image is the same in both cases. Also, differences exist with the focal lengths $f_{x}$ and $f_{y}$ which are not so significant. Since corrected images are in different distances 
(a)

(b)

(c)

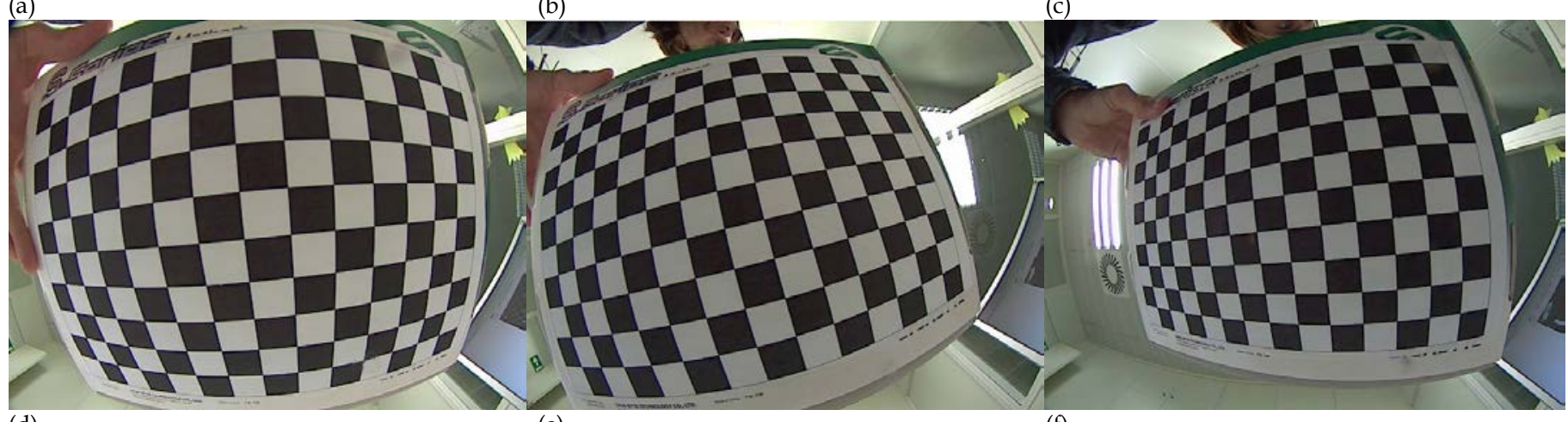

(d)

(e)

(f)

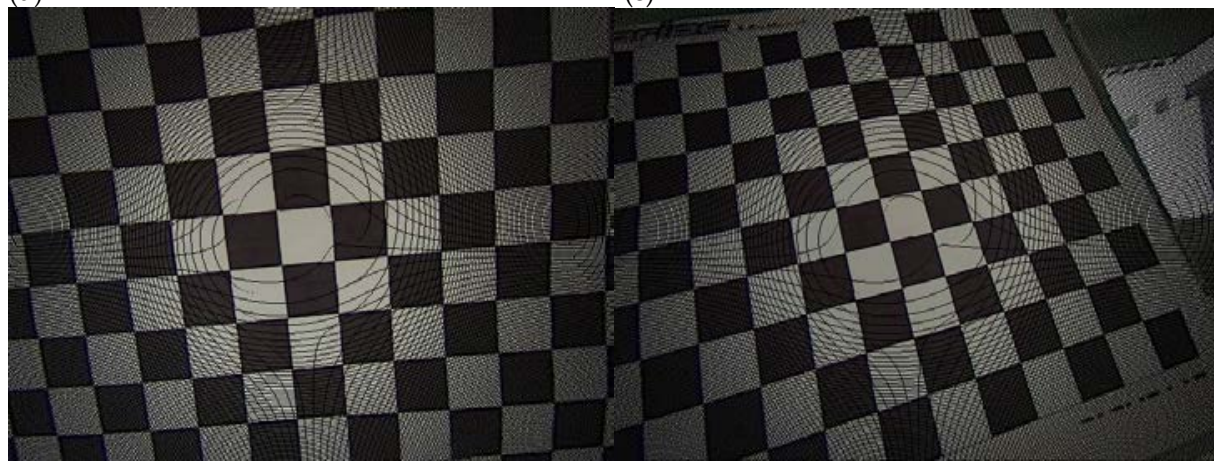

(g)

(h)

(i)

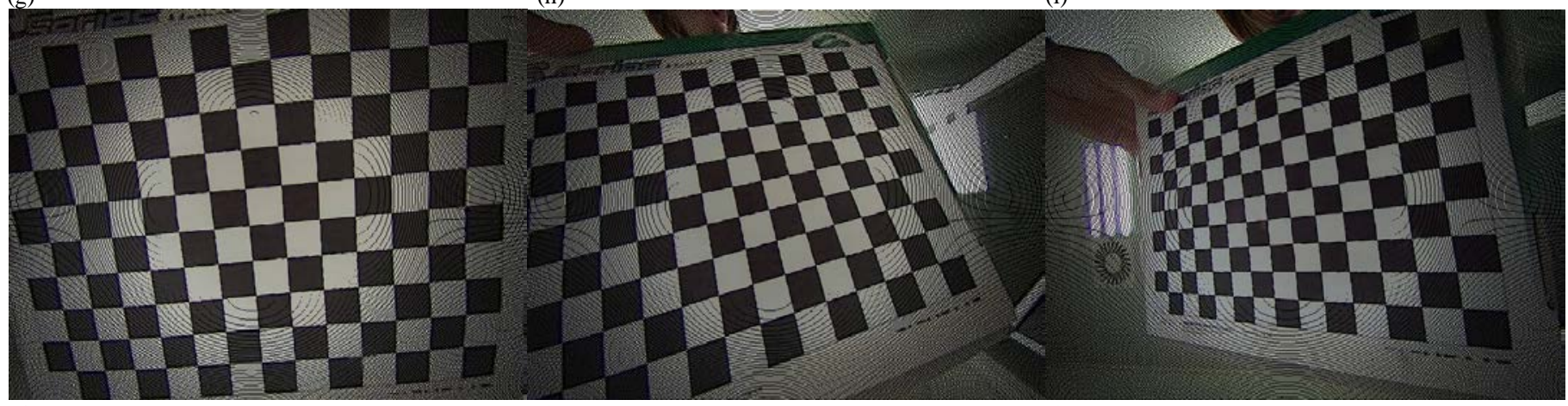

(j)

$(\mathrm{k})$

(l)
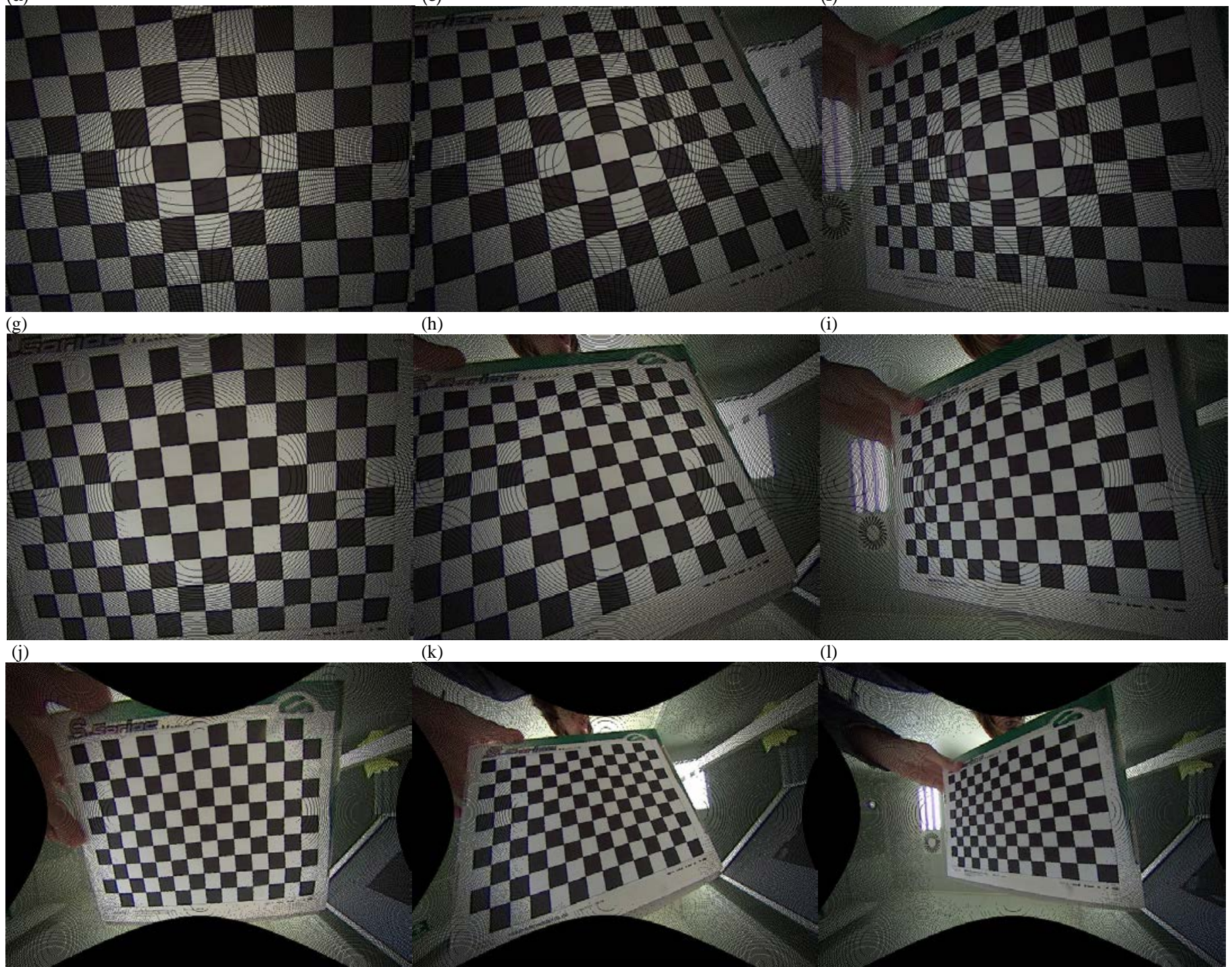

Fig. 5. Results with real data. (a)(b)(c) show 640x480 captured images with an Axis 212 PTZ with $2.7 \mathrm{~mm}$ lens mounted which gives $85^{\circ}$ field of view. (d)(e)(f) show images corrected with the proposed method. (g)(h)(i) show images corrected with the polynomial fish-eye lens distortion correction (PFE) presented by Devernay and Faugeras in [4]. (j)(k)(l) show the results with the non metric calibration of lens distortion (NMC) proposed by Ahmed in [21]. The proposed method does not move the pixels located in the central area of the image.

from the camera, these differences are reflected in the translation in the $\mathrm{Z}$ axe and the focal lengths. Variations in the focal lengths are less significant than variations in the translation in the $\mathrm{Z}$ axe. The correlation between the lens distortion and the pin-hole models influences the translation vector and the lens distortion parameters mainly.

\section{CONCLUSION}

A specific method for calibration of wide angle lens cameras has been defined. One or several images of a planar 
calibration template are taken and pixels locations in distorted images are corrected. Correction is done taking into account that pixels located in the central area of the image should not be moved significantly. When pixels locations are corrected pin-hole and lens distortion models are computed. Distortion model is adjusted to map from points detected in the images to the corrected ones. Pin-hole is computed with corrected points and points from calibration template. This method avoids the correlation between intrinsic, extrinsic and lens distortion models which influences significantly the result of the calibration process, especially in the camera translation vector. To obtain successful results, zenital images of the planar template should be avoided.

\section{REFERENCES}

[1] D. C. Brown, "Close-range camera calibration," Photogrammetric Engineering, vol.37, pp. 855-866, 1971.

[2] J. Weng, P. Cohen, and M. Herniou, "Camera calibration with distortion models and accuracy evaluation,” IEEE Trans. Pattern Analisys Machine Intelligence, vol. 14, pp. 965-980, 1992.

[3] Z. Zhang, "A flexible new technique for camera calibration,” IEEE Trans. Pattern Anal. Machine Intell. Vol. 22, pp. 1330-1334, 2000.

[4] F. Devernay and O. Faugeras, "Straight lines have to be straight," Machine Vision and Applications. Vol. 13, pp. 14-24, 2001.

[5] S. Shah and J. K. Aggarwal, "Intrinsic parameter calibration procedure for a (high distortion) fish-eye lens camera with distortion model and accuracy estimation,” Pattern Recogn. Vol. 29, pp. 1775-1778, 1996.

[6] J. Lavest, M. Viala, and M. Dhome, "Do we really need accurate calibration pattern to achieve a reliable camera calibration," in 1998 Proc. of the European Conference on Computer Vision.

[7] A. Basu and S. Licardie, "Alternative models for fish-eye lenses," Pattern Recogn. Lett. Vol. 16, pp. 433-441, 1995.

[8] A. Fitzgibbon, "Simultaneous linear estimation of multiple view geometry and lens distortion," in 2001 Proceedings of the Conference on Computer Vision and Pattern Recognition, pp. 125-132.

[9] R. Hartley and S. Kang, "Parameter-free radial distortion correction with center of distortion estimation,” IEEE Trans. Pattern Anal. Machine Intell. Vol. 29, pp. 1309-1321, 2007.

[10] D. Claus and A. Fitzgibbon, "A rational function lens distortion model for general cameras," in 2005 Proc. of the International Conference on Computer Vision and Pattern Recognition, pp. 213-219

[11] S.S. Beauchemin, R. Bajcsy, "Modelling and removing radial and tangential distortions in spherical lenses", Multi Image Analysis, Lecture Notes in Computer Science, vol 2023 pp. 1-21, 2001

[12] R. Swaminathan, S. Nayar, "Non metric calibration of wide-angle lenses and polycameras". IEEE Transactions on Pattern Analysis and Machine Intelligence 22 (10) pp. 1172-178, 2002

[13] J. Wang J., F. Shi F., J. Zhang , Y. Liu, "A new calibration model of camera lens distortion”. Pattern Recognition 41 pp. 607-615, 2008

[14] A. Wang, T. Qiu, L. Shao, "A simple method of radial distortion correction with centre of distortion estimation". Journal of Mathematical Imaging and Vision 35: pp. 165-172, 2009

[15] G. Zhang, J. He, X. Yang, “Calibrating camera radial distortion with cross-ratio invariability". Optics and Laser Technology (35) pp. 457461, 2003

[16] D. González-Aguilera, J. Gómez-Lahoz, and P. Rodríguez-Gonzálvez "An Automatic Approach for Radial Lens Distortion Correction From a Single Image”, IEEE Sensors journal, vol. 11, (4) pp 956-966, 2011

[17] P. Sturm, S. Ramalingam, “A generic concept for camera calibration”. Proceedings of the 5th European Conference on Computer Vision, 2004

[18] Z. Zhang, "Camera calibration with one-dimensional objects". IEEE Transactions on Pattern Analysis and Machine Intelligence, 26(7), pp. 892-899, 2004

[19] J. Guillemaunt, A. Aguado, J. Illingworth, "Using points at infinity for decoupling in camera calibration”. IEEE Transactions on Pattern Analysis and Machine Intelligence, 27(2) pp.265-270. 2005
[20] C. Ricolfe-Viala and AJ. Sanchez Salmeron, "Lens distortion models evaluation”, Applied Optics, vol. 49, pp. 5914-5928, 2010.

[21] M. Ahmed, A. Farag, "Non-metric Calibration of Camera Lens Distortion: Differential methods and robust estimation" IEEE Transactions on image processing, vol. 14, no. 8, pp. 1215-1230, 2005

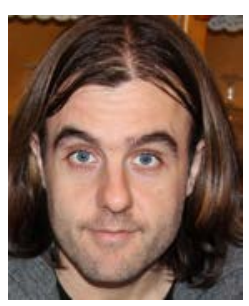

Carlos Ricolfe-Viala: In 1999 he worked one year in the University of Ulm (Germany) as research engineer on computer vision. He received the M.S. degree in 2000 in Electronics and Automatic Control Engineering in the "Universitat Politècnica de València”. In 2006, he obtained his Ph.D. degree on computer vision and its applications. Now he has worked as a lecturer and research scientist in this university since 2000. His research interests are in image processing and computer vision, especially in motion estimation, feature detection and matching, camera calibration, 3D computer vision and intelligent system robot. He also cooperates with the "Instituto de Automatica e Informatica Industrial” (ai2) of Valencia.

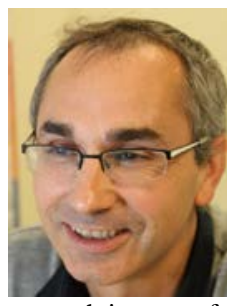

Antonio-Jose Sanchez-Salmeron: He received the BEng in Computer Science degree in 1992, MSc in CAD/CAM/CIM degree in 1999 and Ph.D. degree in Computer Science in 2001. He is currently an Associate Professor at the "Universitat Politècnica de València" (UPV) at the "Departamento de Ingenieria de Sistemas y Automatica" (DISA) and belongs to the Robotics Research Group at the "Instituto de Automatica e Informatica Industrial” (ai2). His research interests focus on the areas of artificial vision and robotics.

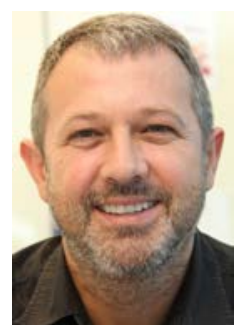

Angel Valera received the B.Eng. degree in computer science, in 1988, the M.Sc. degree in computer science, in 1990, and the Ph.D. degree in control engineering, in 1998, all from the Universitat Politècnica de València (UPV), Valencia, Spain. He has been a professor of automatic control at the UPV since 1989 (Lecturer and Associate Professor), and currently he is a Full Professor in the Department of Systems Engineering and Control at the UPV

He has taken part in research and mobility projects funded by local industries, government, and the European community, and he has authored/coauthored more than 140 technical papers in journals, technical conferences, and seminars. His research interests include industrial and mobile robot control and mechatronics. 